\title{
PREPARACIÓN EN DESASTRES EN EL VEDADO-MALECÓN HABANERO: LA EXPLORACIÓN EN LAS PERCEPCIONES DE SUS LÍDERES
}

\section{Disaster Preparedness Activities in Havana: The Study of the Community Leaders' Perception of Risks}

\section{Preparação em Desastres no Vedado Dique Habanero: A Exploração nas Percepções dos seus Líderes}

Nuria Gaeta Carrillo, PhD, Kampala International University nuriagaetac@gmail.com

Recibido: 14 de abril de 2016

Aceptado: 21 de julio de 2016

\section{RESUMEN}

La reducción de desastres y el aumento de resiliencia de países como meta principal para prevenir desastres no solo requiere de autoridades concienciadas y coordinadas, legislaciones aprobadas y sólidas instituciones. La implicación de las comunidades que los sufren en la medida de tomas preventivas y su preparación para la respuesta es fundamental para la consolidación de comunidades resilientes.

A menudo estas actividades de preparación comunitaria en desastres son implementadas sin un análisis profundo sobre las percepciones, creencias e intereses de sus interlocutores, lo que puede derivar en el fracaso de la intervención. 
A partir de entrevistas individuales, grupales y encuestas, la presente publicación trata de explorar las percepciones de los líderes del Consejo Popular Vedado-Malecón de La Habana que conducen a un comportamiento favorable hacia la reducción del riesgo o hacia uno inadecuado. Esta información podría arrojar luz en el diseño de estrategias de preparación para la reducción del riesgo en el nivel comunitario estudiado.

Palabras clave: preparación en desastres, líderes comunitarios, percepción del riesgo, medidas preventivas, Cuba.

\section{ABSTRACT}

Risk reduction and build resilience in order to prevent some disasters require not just well coordinated authorities, a sound legislation and strong institutions. It is also vital to involve the local communities in preventive measures.

The design of community training and community based preparedness activities is not often planned properly and is done without enough information, leading to a breakdown in the intervention.

Based on personal and group interviews and a survey, this study performs an exploration of community leaders perceptions about risks in Havana that strengthens or constrains preventive measures and enhance or not response capacities. Information that helps to design capacity building activities at studied community.

Keywords: Disaster preparedness, community leaders, risk perception, preventive measures, Cuba.

\section{RESUMO}

A redução de desastres e o aumento de resiliência de países como objetivo principal para prevenir desastres, não só requere de autoridades sensibilizadas e coordenadas, legislações aprovadas e sólidas instituições. A implicação das comunidades que os sofrem na medida de tomas preventivas e a sua preparação para a resposta é fundamental para a consolidação de comunidades resilientes.

Com frequência estas atividades de preparação comunitária em desastres são implementadas sem uma análise profunda sobre as percepções, crenças e interesses dos seus interlocutores, podendo derivar no fracasso da intervenção.

A partir de entrevistas individuais, grupais e enquetes, a presente publicação tenta explorar as percepções dos líderes do Conselho Popular Vedado Dique da Havana que conduzem a um comportamento favorável à redução do risco ou inadequada informação que conseguira lançar alguma luz no desenho de estratégias de preparação para a redução do risco no nível comunitário estudado.

Palavras-chave: preparação em desastres, líderes comunitários, perceção do risco, medidas preventivas, Cuba. 


\section{Introducción}

Durante la década de los setenta del siglo pasado, corregir el conocimiento errado de la población ante un problema dado suponía para los expertos y gobiernos la manera más eficaz de influir directamente sobre las percepciones de las personas, moldeando así las actitudes de estas hacia sus propios intereses. Este planteamiento soslayaba las percepciones que constituyen fenómenos complejos que no solo responden a motivos técnicos, sino también a valores, creencias y otros factores como el miedo. Carmen Almaguer (2008, pp. 44 y ss.) define la percepción como el proceso activo mediante el cual el individuo recoge información de su entorno, la procesa siguiendo criterios culturales, sociales y económicos, y se predispone para la actuación. Indagar en el mundo simbólico de las comunidades (Hollander, 1967), en lo que se refiere al riesgo y a la percepción del riesgo, solo es posible desde dentro del contexto geográfico, dado que se produce y se modifica conforme se interviene en el espacio. Pero, junto al contexto geográfico, no se puede dejar de lado la vulnerabilidad local y el tejido social, añade Almaguer (2008, pp. 44 y ss.).

Siguiendo esta perspectiva, el objetivo de la presente publicación es aproximarse a la cotidianidad de los líderes comunitarios del Consejo Popular Vedado-Malecón ${ }^{1}$ de Ciudad de La Habana, con el fin de conocer sus condiciones de vida, su contexto geográfico, su cotidianidad, la vulnerabilidad social, sus creencias sobre desastres, los conocimientos y actitud hacia la toma de medidas preventivas, para explorar su percepción social sobre riesgos de desastres de origen hidrometeorológicos extremos ${ }^{2}$. Determinar estos factores permite identificar sus necesidades comunicativas ${ }^{3}$ con el fin de diseñar estrategias de preparación comunitaria (Navarro, 2009) para desastres acorde a los intereses y creencias de la población estudiada que generen un comportamiento favorable hacia la reducción del riesgo de desastres (RRD); además de un acercamiento a la problemática desde la sensibilidad y la empatía de la población.

Los objetivos específicos que darán respuesta a dicho objetivo son:

1. Identificar sus elementos de vulnerabilidad social y ambiental.

2. Determinar las vivencias y experiencias de la comunidad en emergencias y desastres anteriores.

3. Caracterizar los actores comunitarios que median los procesos de prevención de desastres en la comunidad estudiada.

1 Uno de los barrios de Ciudad de La Habana más afectado por desastres de origen hidrometeorológico extremos (Mitrani, 2000).

2 Un grave trastorno desencadenado por ciclones tropicales o tormentas locales severas que provocan pérdidas humanas, materiales, económicas y/o ambientales y que exceden la capacidad de los afectados de lidiar con ellas (definición construida a partir de los conceptos presentes en la Directiva №1 de 2005).

3 Se refieren a las lagunas informativas, déficit de conocimiento, intereses informativos o comunicacionales o percepción del riesgo de la población en materia de prevención de desastres o diagnóstico situacionales o de la comunicación (Baccin, 2001). 
4. Indagar en el conocimiento, interés y valores de los actores receptores de los procesos de prevención de desastres en la comunidad.

5. Determinar las formas de resolver el riesgo de manera preventiva en la práctica o en su imaginación.

Esta publicación supone una parte de los resultados de la tesis doctoral titulada La intervención comunicativa para la Reducción del Riesgo de desastres. Análisis de las políticas y las prácticas en tiempos normales en Cuba, defendida en la Universidad Complutense de Madrid en diciembre de 2015.

\section{Antecedentes}

Las intervenciones para la RRD deben ser proactivas y permanentes (PNUD, 2004). Con esta premisa de partida, la etapa del ciclo de reducción de riesgos de desastres ${ }^{4}$ que incluye un mayor número de intervenciones proactivas, supone un mayor compromiso político y un nivel de maduración lógica más sustantivo en lo correspondiente a la prevención. Las acciones desarrolladas durante la etapa preventiva son las únicas que coadyuvan a la consecución de transformaciones profundas vinculadas con la reducción del riesgo de desastre, la reducción de vulnerabilidades (Beltrán, 2004) y el aumento de la resiliencia. La adopción de medidas preventivas en el nivel comunitario, así como la movilización y la respuesta durante emergencias, dependen en gran medida ${ }^{5}$ de la percepción del riesgo y el conocimiento sobre este.

En los últimos 25 años, la lógica que guiaba las intervenciones de reducción del riesgo de desastres ha evolucionado, así como los conceptos rectores en la preparación para desastres. Aunque el enfoque social ha sido el que en mayor medida se ha encargado de los estudios de percepción social, los conceptos que aportó la técnica y que guían principalmente la RRD -en los niveles nacional e internacional- justifican las acciones aquí estudiadas.

Desde la perspectiva técnica, se entiende que se produce un desastre cuando se da un impacto medible en la sociedad, el medio ambiente y/o la economía (Almaguer, 2008, p.10). Gracias a este enfoque, el riesgo de desastre se comenzó a definir en función del peligro ${ }^{6} \mathrm{y}$ de la vulnerabilidad ${ }^{7}$. Para esta perspectiva, ya que sobre la amenaza

4 El Decreto Ley n¹70 de 1997 de Cuba define la reducción de riesgo de desastre como el "conjunto de actividades preventivas, de preparación, respuesta y recuperación que se establecen con la finalidad de proteger a la población, la economía y el medio ambiente de los efectos destructivos de los desastres naturales u otro tipo de catástrofes".

5 Otro elemento fundamental que, aunque no es objeto de estudio, aparece durante la investigación es la confianza entre actores (López y Luján, 2010; Gaeta y Lorenzo, 2012).

6 Peligro según la legislación cubana es "Un fenómeno (de origen natural, tecnológico o sanitario) que puede provocar una alteración altamente dañina en el orden público" (Cuba, 2005).

7 Según la Estrategia Internacional de Reducción de Desastres la vulnerabilidad es "el conjunto de situaciones personales y sociales que dejan a las personas frágiles e indefensas ante la ocurrencia de una amenaza o peligro" (UNISDR, 2009). 
no se puede intervenir directamente ${ }^{8}$, la reducción de desastres supone la reducción de vulnerabilidades ${ }^{9}$, concepto rector durante el cambio de siglo. Desde la preparación para desastres (Beltrán, 2004) este enfoque supone crear conciencia ${ }^{10}$ sobre los riesgos, así como generar una percepción social de los riesgos adecuada.

Hoy día, el contexto internacional de reducción de desastres promueve el aumento de resiliencia de las comunidades que, según la Estrategia Internacional de Reducción de Desastres, es "la capacidad de un sistema, comunidad o sociedad expuestos a una amenaza para resistir, absorber, adaptarse y recuperarse de sus efectos de manera oportuna y eficaz, lo que incluye la preservación y la restauración de sus estructuras y funciones básicas" (UNISDR, 2009). La preparación para desastres ${ }^{11}$ coadyuva a aumentar la resiliencia de las comunidades, en la medida en que la población no es solo consciente de los riesgos, sino que desarrolla además herramientas para resistir, absorber, adaptarse y recuperarse de los impactos de las amenazas.

En cuanto a la perspectiva social, desde los estudios de White (1945), esta ha puesto el acento en las causas humanas de los desastres y ha enfatizado la idea de que la percepción social de los peligros influye en la toma de decisiones de las comunidades para que su medio sea más seguro; lo cual deviene en la introducción de un elemento clave a la hora de reducir el riesgo de desastre: lo cultural. Para las nuevas corrientes sociológicas, el riesgo se define como construcciones sociales que dependen de factores socioculturales vinculados a estructuras sociales dadas (Luhmann, 1998; López y Luján, 2000). Hay que destacar los aportes de cinco campos conceptuales, de carácter transversal, que han contribuido desde las ciencias sociales a un mayor conocimiento del riesgo en términos de su proceso de construcción, según Althaus (Farré y Gonzalo, 2009, p. 120). Estos campos son: la percepción, la confianza, la incertidumbre (López y Luján, 2000), la gobernabilidad y la comunicación. Farré y Gonzalo (2009, p. 121) parten de la percepción del riesgo como primer escalón en su construcción, y desembocan en el proceso de comunicación, incorporando entre medias los estadios de la confianza, la incertidumbre y la gobernabilidad. Existe una compleja relación - advierten los autores- según la cual, para obtener conocimiento sobre la percepción del riesgo, se debe intervenir en los mecanismos de construcción de la confianza que, a su vez, se encuentran interferidos por la exigencia de la gestión de la incertidumbre y del reconocimiento en el reparto de responsabilidades conducente hacia la gobernabilidad del riesgo a través del diálogo social y democrático.

8 Numerosos autores (Wilches-Chaux, 1989, 2011; Allan Lavell, 1996, 2000; e incluso las Naciones Unidas, PNUD, 2004) defienden la intervención sobre la amenaza, por ejemplo, reducir las emisiones de gases invernadero que contribuye a reducir el calentamiento global.

9 Para ampliar la información en las formas de reducir la vulnerabilidad ver Castellanos (2009) o Beltrán (2004). La intervención sobre la vulnerabilidad comprende entre otras operaciones reubicación de las comunidades asentadas en zonas de riesgo; reforestación de cuencas y laderas; empleo de sistemas constructivos y antisísmicos y defendibles de inundaciones; instalación de sistemas permanentes de monitoreo de amenazas, vulnerabilidad y riesgos; y formulación de códigos de instrucción (Beltrán, 2004).

10 Entendida como la capacidad de tomar decisiones a partir de un racional acto reflexivo, fundamentado en la responsabilidad y la actuación consciente y consecuente de lo que se siente, se piensa y se conoce, en función de una meta a lograr (Gaeta, 2015).

11 Trata de evitar o minimizar las pérdidas de vidas humanas y daños materiales, formando a las instituciones y a la población (UNISDR, 2009). 
Partiendo de dichos antecedentes, este estudio tratará de acercarse a algunos rasgos psicológicos, psicosociales o ambientales de los sujetos - propios de los enfoques piscométricos (Slovic, 1981, 2002; Fischhoff, 1995) en los estudios de percepción-. También tendrá en cuenta lo que la gente dice y siente, al escuchar y dar valor a sus expresiones verbales y gestuales, el lenguaje empleado y las prácticas sociales en donde se inscribe el sujeto de estudio, propio de los métodos cualitativos (Balavnosky, Redin y Pogiesse, 2001; Puertas, 2003); así como la actitud hacia la toma de medidas preventivas ${ }^{12}$, con especial dedicación a la predisposición de la población para evacuar (Gaeta y Lorenzo, 2010), a fin de acercarse a la percepción y a las necesidades comunicativas de la población objeto de estudio como primer eslabón de la construcción del riesgo.

\section{Metodología}

No es de extrañar que, con estos antecedentes teóricos, se escogiera el estudio de caso único como método investigativo, por ser el método que probablemente permite un mayor acercamiento a la vida de la comunidad escogida, facilitando el entendimiento de sus creencias, mitos y ritos sobre la temática estudiada.

Tras un largo proceso burocrático para conseguir las cartas pertinentes que autorizaran el correcto desarrollo de la investigación y a la par que se ahondaba en el análisis documental - principalmente para los aspectos evolutivos referidos a políticas y al modelo de gestión del riesgo en Cuba - se desarrollaron entrevistas individuales y grupales, se aplicó un cuestionario y se empleó la observación sin participación como técnicas de acopio de la información.

Mediante entrevistas individuales, se indagó en los conceptos básicos que sustentan la preparación para la reducción del riesgo en la localidad estudiada, sirvió también para identificar el rol de los principales actores estratégicos en la RRD, la confianza entre actores y comunidad y la metodología empleada, además de captar otras opiniones de especialistas y técnicos. Se realizaron un total de 20 entrevistas (12 entrevistas en profundidad y 8 semiestructuradas) a expertos en desastres, comunicólogos, comunicadores y actores ${ }^{13}$ estratégicos vinculados con la RRD.

Se aplicó un cuestionario a los líderes informales de la zona objeto de estudio que permitió indagar en sus creencias, conocimiento, actitud hacia la toma de medidas preventivas ${ }^{14}$, entre otras cuestiones. La muestra del cuestionario se determinó contactando con todos los presidentes de los $\operatorname{CDR}^{15}$ objeto de estudio (13 en total), y siguiendo criterios de viabilidad se les pidió que identificaran a tres personas con cualidades de líder ${ }^{16}$ en su correspondiente manzana/CDR. Un total de 42 líderes informales participaron en el cuestionario. La selección de la

12 Especialmente, la predisposición a evacuar que supone la medida preventiva más significativa en casos de severas amenazas (Gaeta y Lorenzo, 2010).

13 En esta amplia categoría se incluyen a agentes que trazan, influyen o supervisan políticas de reducción del riesgo, comunicadores, gobierno local, dirigentes de organizaciones políticas y de masas y líderes formales comunitarios.

14 A partir del documento "Mitos y realidades sobre desastres en Cuba" (VV.AA, s/f).

15 Los presidentes de CDR son líderes formales.

16 Vecinos de la manzana que por su carácter, comportamiento, nivel económico o inteligencia, responsabilidad, empatía y constancia en las labores comunitarias entre otros factores posean credibilidad entre el resto de la población, pudiendo convertirse en formadores, guías y movilizadores de otros vecinos de la cuadra. 
muestra supuso prácticamente el $100 \%$ de los líderes informales del área objeto de estudio pues, según varios presidentes consultados, resultó una tarea complicada identificar en sus manzanas a tres líderes informales.

La entrevista grupal, en la que participaron los 13 dirigentes locales correspondientes a cada CDR, supuso un acercamiento cualitativo a los líderes formales de la comunidad. Para su ejecución se contó con el apoyo del delegado de circunscripción que diseminó la convocatoria. La técnica se desarrolló entre la investigadora en su rol de moderadora y un observador silencioso que anotaba los comentarios de los participantes, así como la información de otra índole referida a las expresiones, actitudes, posturas y confrontaciones que surgieron con respecto al objeto de estudio. El desarrollo de la dinámica se realizó de acuerdo con una guía predeterminada que marcaba los contenidos y el tiempo dedicado a cada bloque temático.

Finalmente, se obtuvo información directa a través de la observación sin participación en espacios públicos (cinco) y cerrados (dos) que permitió verificar o contrastar la información obtenida a través de otras técnicas.

\section{Resultados}

\section{Vulnerabilidades, riesgos y amenazas en el Consejo Popular Vedado-Malecón}

El rasgo físico más característico de la circunscripción objeto de estudio es que linda al norte con el mar Atlántico, lo que condiciona inexorablemente la vida de la vecindad. Su tramo costero está protegido por un muro de hormigón -el emblemático Malecón habanero-que de alguna manera protege el barrio de los embates del oleaje y está bordeado por una ancha avenida escoltada por altas edificaciones.

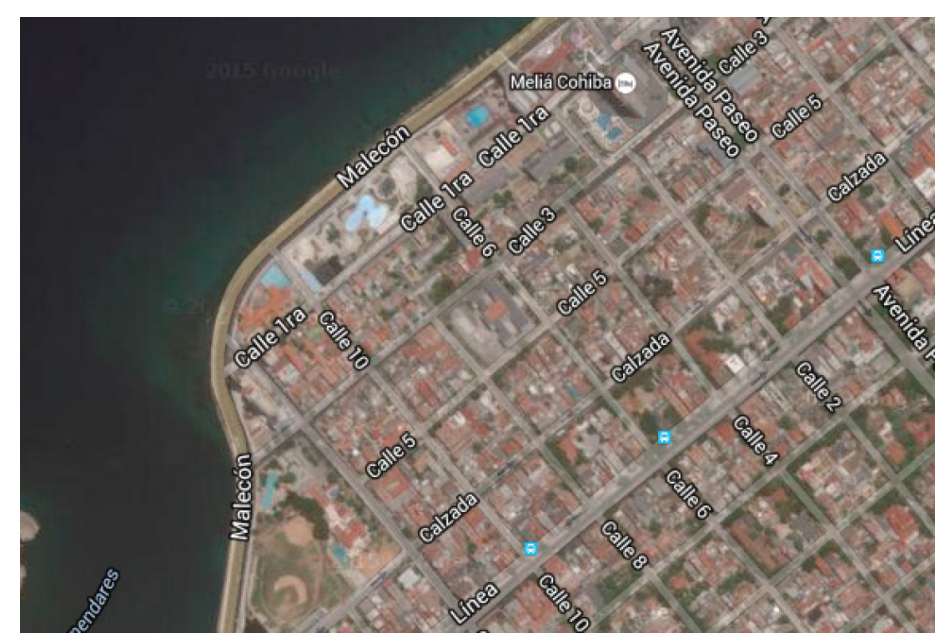

El Consejo sufre inundaciones causadas por penetraciones de mar y fuertes lluvias que afectan directamente a más del $60 \%$ de la población e indirectamente al $100 \%$ de ella. Concretamente, el tramo donde se localiza la zona objeto de estudio posee una terraza costera de 20 a 160 metros de ancho con una pendiente muy suave y con la más alta incidencia de penetraciones del mar en el territorio nacional. 
La circunscripción № 62 está amenazada durante los 12 meses del año por riesgo de inundaciones debido a su ubicación dentro del área donde se produce el llamado "efecto palangana"17. Las inundaciones ${ }^{18}$ tienen una $^{17}$ frecuencia media de dos por año, según Liudmila Labrada, del Taller de Transformación Integral del Barrio ${ }^{19}$.

La circunscripción estudiada posee además una serie de características que la hace especialmente vulnerable al impacto de los eventos hidrometeorológicos extremos (Caballero, 2010):

- Cuenta con un mayor número de ciudadelas, las cuales están asociadas a serias problemáticas socioeconómicas.

- Es la circunscripción en peor estado constructivo.

- Superpoblación en algunas manzanas.

- Acusada deforestación en la parte del Malecón.

- Limitaciones de agua.

- Problemas de alcantarillado.

- Acusadas desigualdades en las necesidades de la población.

- Su ubicación espacial dentro del área donde se produce el "efecto palangana".

Los principales problemas detectados por los trabajadores sociales en relación a la respuesta de desastres son:

- El incumplimiento y desobediencia de las orientaciones de la Defensa Civil en cuanto a las medidas para la salud y el resguardo de bienes personales.

- La ausencia de un plan completamente efectivo.

\section{Experiencia en emergencias anteriores}

Existen dos experiencias que han marcaron notablemente la vida de sus pobladores en relación a los desastres: la “tormenta del siglo" en 1993, y el huracán Wilma en 2005.

El 13 de marzo de 1993 tuvo lugar la llamada "tormenta del siglo", "que prácticamente dejó todo desolado, desde el punto de vista de los bienes materiales", explica Xiomara Leyva (exdelegada de la circunscripción), lo que impulsó la creación de mecanismos para reducir el riesgo de desastres.

Otro antecedente importante fueron las inundaciones provocadas por el huracán Wilma, el 20 de octubre de 2005. En esta ocasión, narra Leyva Matos, del gobierno del municipio, el problema principal fue la inapropiada valoración de la población, aún así las estructuras ya estaban creadas y las pérdidas fueron mínimas en comparación a 1993. "Con la experiencia del 93, muchas personas se confiaron e hicieron construcciones internas en sus casas para subir los equipos, los muebles, etcétera, teniendo en cuenta los límites marcados durante aquellas inundaciones" cuenta Xiomara, pero el Wilma sobrepasó esos límites.

17 Acumulación de agua producida por inundaciones, tanto del agua que penetra del mar, como del que cae de la colina de El Vedado cuando se producen lluvias intensas.

18 Provocadas por fuertes lluvias o penetraciones de mar las cuales, a su vez, son producidos por frentes fríos, bajas extratropicales, ciclones y huracanes (Mitrani, 2000).

19 Actor comunitario estratégico en labores de reducción de riesgo. 
A pesar del aprendizaje de estas dos experiencias, la circunscripción sufre inundaciones anuales sin que el problema se haya resuelto todavía.

No es de extrañar que con las características descritas anteriormente -azote constante de ciclones y frentes fríos, inundaciones frecuentes, deterioro habitacional, hacinamiento, deforestación, delincuencia...- los líderes comunitarios cumplan una misión encomiable en el trabajo de esta localidad. Se exponen a continuación las principales características de los líderes formales como diseminadores de los planes de reducción del riesgo en el nivel comunitario estudiado y de los líderes informales como su principales interlocutores y aliados estratégicos.

\section{Líderes formales: tipología y caracterización}

A partir de la información obtenida en las entrevistas, se define a los líderes formales en el área estudiada como un tipo particular de aliados estratégicos, nombrados formalmente para la representación y el cuidado de la comunidad. Son los máximos responsables comunitarios de hacer llegar las directrices marcadas en los planes nacionales y, por consiguiente, cumplen una misión fundamental como diseminadores y socializadores de las políticas de RRD en las comunidades. Son vecinos del barrio, lo cual los convierte en excelentes conocedores de las problemáticas locales.

Los líderes formales se puede clasificar entre aquellos que de manera voluntaria dedican su tiempo libre a tareas comunitarias (encargados de organizaciones de masas y políticas y otras estructuras comunitarias) y los que trabajan con la comunidad, como el personal sanitario, educativo o los trabajadores sociales. Los líderes formales comunitarios detectados en la zona objeto de estudio ${ }^{20}$ son: el Comité de Defensa de la Revolución (CDR), la Federación de Mujeres Cubanas (FMC), los Talleres de Transformación Integral del Barrio (TTIB), los trabajadores sociales y el personal sanitario ${ }^{21}$.

La mayoría de los sujetos entrevistados tiene un nivel académico medio alto -licenciados o técnicos medios$y$, en general, posee un vasto conocimiento acerca de qué es un desastres y qué medidas se deben tomar en caso de inundaciones, resultado de la propia experiencia empírica y las sesiones formativas que organiza el municipio.

Se observaron, en algunos casos, deficiencias comunicativas necesarias para asumir el rol de facilitadores de la información: dificultades para expresarse; una dedicación superficial a contenidos que demandan explicaciones profundas; y una jerarquía de los temas expuestos inadecuada. No obstante, a pesar de estas limitaciones, la cercanía que mantienen con la comunidad y el conocimiento del tema que se aborda salvaguarda el proceso comunicativo mediante la explicación sencilla pero clara de las ideas principales, un lenguaje extraverbal suavizado en sus formas y la respuesta a los interrogantes de la comunidad.

La mayoría de los actores consultados ${ }^{22}$ considera la prevención de desastres un factor imprescindible en la reducción del riesgo que demanda de una preparación permanente de la población. En este proceso de capaci-

20 Queda aquí constancia del importante rol de los educadores y los directores de escuela. Actores que no forman parte del estudio debido a la falta de autorización para su investigación.

21 Para ampliar la información sobre su organización y funciones ver Gaeta (2016).

22 Aunque en menor proporción, hay también líderes que consideran que el abuso de prácticas educativas de RRD pueden provocar el efecto deseado contrario. 
tación "la comunicación es la base fundamental para desarrollar este trabajo en la población", afirma Ana María Álvarez, coordinadora de CDR de la zona.

Los líderes formales comunitarios organizan reuniones de vecinos donde se tratan los problemas de carácter colectivo; participan en el ejercicio nacional de entrenamiento para situaciones de desastres (llamado Meteoro) y en los Días de la Defensa ${ }^{23}$, cuyos contenidos son impuestos por el municipio.

Anualmente, los municipios actualizan las indicaciones para la preparación para desastres ${ }^{24}$ a partir del aporte de los CDR y de la Federación de Mujeres Cubanas. Sin embargo la entrevista grupal reveló el descontento existente entre los entrevistados debido a la escasa adecuación de los contenidos, con la problemática de cada cuadra, probablemente causa de la heterogeneidad socioeconómica subyacente.

Por unanimidad, los presidentes de CDR consultados identifican como el principal problema que sufre la zona el deterioro constructivo del fondo habitacional ${ }^{25}$, el cual supone causa y consecuencia de múltiples desastres. Sin embargo, hay desacuerdo en la forma de resolver dicha problemática. Las propuestas oscilan desde posturas que procuran una reparación sostenible a largo plazo que visualiza el proceso resolutivo como parte de la solución del problema al generar, por ejemplo, una fuente de empleo ${ }^{26}$ a los vecinos del Consejo, hasta otras no formales que buscan la inmediatez en los arreglos mediante la construcción de muretes tipo diques, vallas y otras alternativas transitorias de alto impacto en la imagen urbana, traspasando en ocasiones lo contemplado por las regulaciones urbanas vigentes y dejando de aplicar una lógica de reducción de vulnerabilidades a largo plazo. Esta solución, con miras a resolver el problema a corto plazo, es propuesta sobre todo por aquellos que sufren directamente el mal estado constructivo.
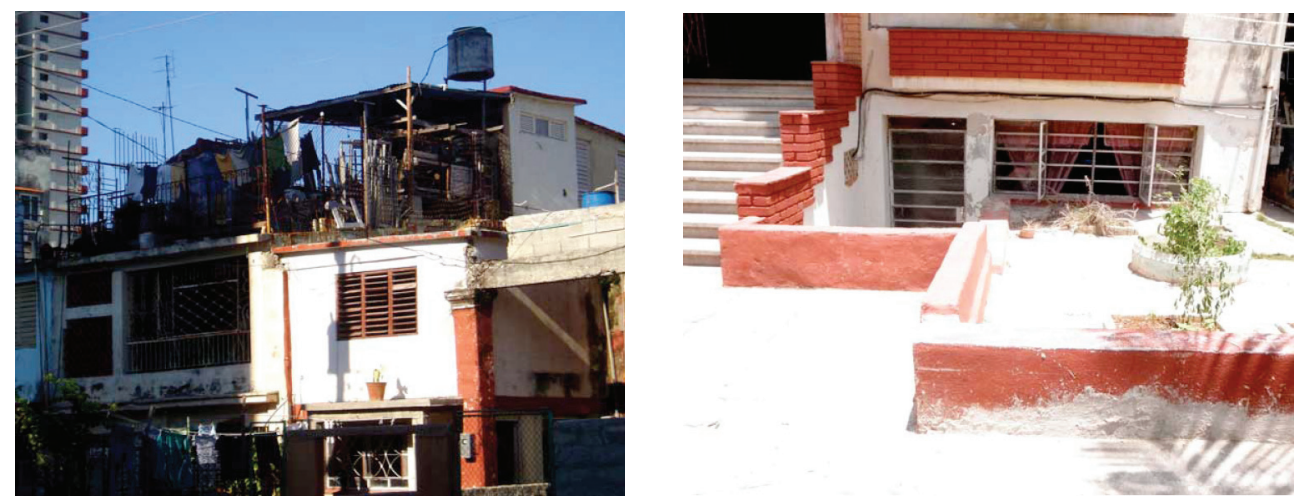

División de viviendas y viviendas por debajo del nivel de la calle son algunos de los problemas a los que se enfrenta la localidad

23 Por ley, se establecen tres días de la defensa anualmente. En estos se organizan charlas y talleres sobre cuestiones referidas a diversas problemáticas que afectan a los municipios.

24 Las Indicaciones Metodológicas de Organización.

25 La realidad descrita está determinada, en parte, por el problema habitacional que vive la ciudad y que provoca el hacinamiento de familias en hogares y fomenta las construcciones irregulares.

26 Liudmila Labrada, del Taller de Transformación del Barrio comentaba: "hay muchas ciudadelas en este área que se inundan [...] y quizás esa transformación pudiera ser una fuente de empleo para la misma población que vive dentro de este Consejo". 


\section{Los líderes informales: creencias y actitudes}

En la investigación se trabajó la intervención comunicativa sobre los actores de la comunidad con cualidades de líderes informales ${ }^{27}$ por su potencial como facilitadores y transmisores de la información.

\section{Caracterización de los líderes informales}

A pesar de la heterogeneidad en la comunidad estudiada, las características sociodemográficas y culturales de los líderes informales se repiten prácticamente en la totalidad de la muestra.

En cuanto a las diferencias por sexo llama la atención que, al ser en su mayoría hombres los presidentes de CDR (líderes formales), estos identifican principalmente a la mujer como líder informal (69,9\%) al apelar a su carisma, inteligencia, nivel de responsabilidad e implicación con el CDR, entre otros motivos. Además, se da la relación proporcional que a mayor edad, mayor nivel de participación y compromiso con la comunidad. Los líderes informales pertenecen en su mayoría al grupo etario representado por la población de 59 a 74 años que supone un 45,23 \% de la muestra. En oposición, el grupo que menos participa en la vida comunitaria son los jóvenes con edades comprendidas entre 18 y 29 años, con un 16,6 \%. El nivel educativo de los líderes informales es medio y medio alto, sin existir apenas diferencias por sexo.

Un dato significativo es el tiempo en que los nuevos habitantes de la comunidad empiezan a involucrase en las problemáticas comunitarias. El estudio revela que a partir de los tres años de habitar en el mismo territorio, comienzan a brotar sentimientos de arraigo y se van forjando los nuevos líderes comunitarios. El 76,19 \% de los líderes informales de la muestra lleva viviendo entre tres y diez años en su lugar de residencia (ver tabla 1).

\section{Tabla 1: Individuos según sexo y tiempo de residencia en El Vedado-Malecón}

\begin{tabular}{|l|l|l|l|}
\hline \multicolumn{1}{|c|}{ Sexo / Tiempo residencia } & \multicolumn{1}{c|}{ Menos 3} & \multicolumn{1}{c|}{ De 3 a 10} & \multicolumn{1}{c|}{ Más de 10} \\
\hline Masculino & $11,9 \%$ & $26,19 \%$ & $0 \%$ \\
\hline Femenino & $9,52 \%$ & $50 \%$ & $2,38 \%$ \\
\hline
\end{tabular}

Fuente: Elaboración propia a partir de los datos del cuestionario.

En el área estudiada, el 80,95 \% de la muestra coincide en perfilar los límites de su comunidad en un ámbito superior al del hogar o la familia, lo que constituye una concepción solidaria y de cooperación de la vecindad que favorece el trabajo comunitario. Idea compartida por los presidentes de CDR y los trabajadores comunitarios que afirman que la cooperación entre vecinos es incluso más profunda en casos de emergencia, situación que responde a lo que autores como Núñez (s.f, p. 90) han denominado la "pedagogía del desastre".

27 Según Xiomara Leyva, delegada de la zona durante más de 20 años, los líderes informales son "vecinos como otros cualquiera que han ido asumiendo o han aceptado la comisión de prevención de la cuadra". 


\section{Niveles de concienciación en prevención de desastres}

En la localidad estudiada, el $100 \%$ de los entrevistados ${ }^{28}$ coincide en afirmar que los vecinos del Consejo tienen un nivel de concienciación elevado. Citando algunos ejemplos, Liudmila Labrada, del Taller de Transformación, afirma que "ellos saben que tienen que estar preparados todo el tiempo"; Ana María Álvarez, coordinadora de CDR de la Circunscripción № 62, confirma que "sí, la mayoría lo sabe"; Inola Díaz, autora del Programa de Comunicación para Desastres (2006) sostiene que "en general conocen bastante, no sé si todos pero sí bastante”; y Mark Ingelbrecht, técnico en desastres de la ONG Oxfam en Cuba, admite que "el nivel de conciencia que hay en la población es realmente elevado, todo el mundo sabe quién es el referente a la hora de que algo va a pasar".

Las respuestas de los líderes informales a la pregunta qué riesgos suponen una mayor amenaza para su comunidad (ver gráfico 1), cruzados con los buenos resultados obtenidos cuando un ciclón, huracán u otro evento hidrometorológico atraviesa el país (Oxfam América, 2004; Oxfam Solidaridad, 2008), corroboran la convicción expresada por el resto de actores sociales. La categoría "inundaciones" fue el riesgo más reconocido por la muestra con un $66,6 \%$ de respuestas a su favor, tal y como lo identifica la Directiva № 1 para Desastres y otros informes sobre vulnerabilidad del país y de la ciudad ${ }^{29}$.

Tras las inundaciones, los vecinos estudiados identifican otro tipo de eventos que se muestran en un equilibrio de prioridad, como interrupciones de electricidad (30,95\%) o derrumbes de edificios frágiles (30,95\%). El riesgo de inundaciones durante los 12 meses del año y el deterioro habitacional son problemas interrelacionados, detectados y reconocidos por todos los actores sociales de la zona.

No cabe duda de que la experiencia empírica que cada persona ha sufrido en emergencias anteriores influye poderosamente en el nivel de conciencia sobre el riesgo, el conocimiento de las formas de resolverlo y la percepción de este que instiga, aunque no determina, la toma de medidas preventivas. Por ello, para algunos líderes comunitarios, los vecinos de El Vedado-Malecón son conscientes de los riesgos que corren desde 1993, año en que "sufrieron una gran sacudida", afirma Xiomara Leyva. La totalidad de las personas consultadas posee experiencia en eventos hidrometeorológicos extremos, lo que aumenta su nivel de conciencia y contribuye a la toma de medidas preventivas en su favor.

\section{Factores que limitan la capacidad preventiva y de respuesta de los líderes informales}

La leve penetración de mar que vivió el barrio el 3 de marzo de 2010 sorprendió a muchos. A Ana María, la coordinadora de CDR, que vivió las inundaciones de 1993 y de 2005, la inundación la afectó tan de repente, que no pudo seguir con las tareas de organización y ayuda en la evacuación. En su relato narra que "[...] ayudando a evacuar a los vecinos y sus bienes, cuando subí a mi casa para cambiarme los zapatos, ya no pude bajar más porque una sola ola había inundado todo, nunca había visto llegar a ese nivel". El factor sorpresa siempre reaparece en situaciones de emergencia, aun cuando se tenga una vasta experiencia y conocimiento sobre desastres.

28 Decisores políticos, expertos en desastres, trabajadores y técnicos en gestión de reducción de desastres y líderes comunitarios

29 Conviene recordar que el caso más significativo en cuanto a la combinación de peligro, vulnerabilidad y riesgo resultante de las penetraciones de mar en el país lo constituye el Malecón habanero (Rodríguez, 2009). 
Gráfico 1. Identificación de riesgos y amenazas de los líderes informales

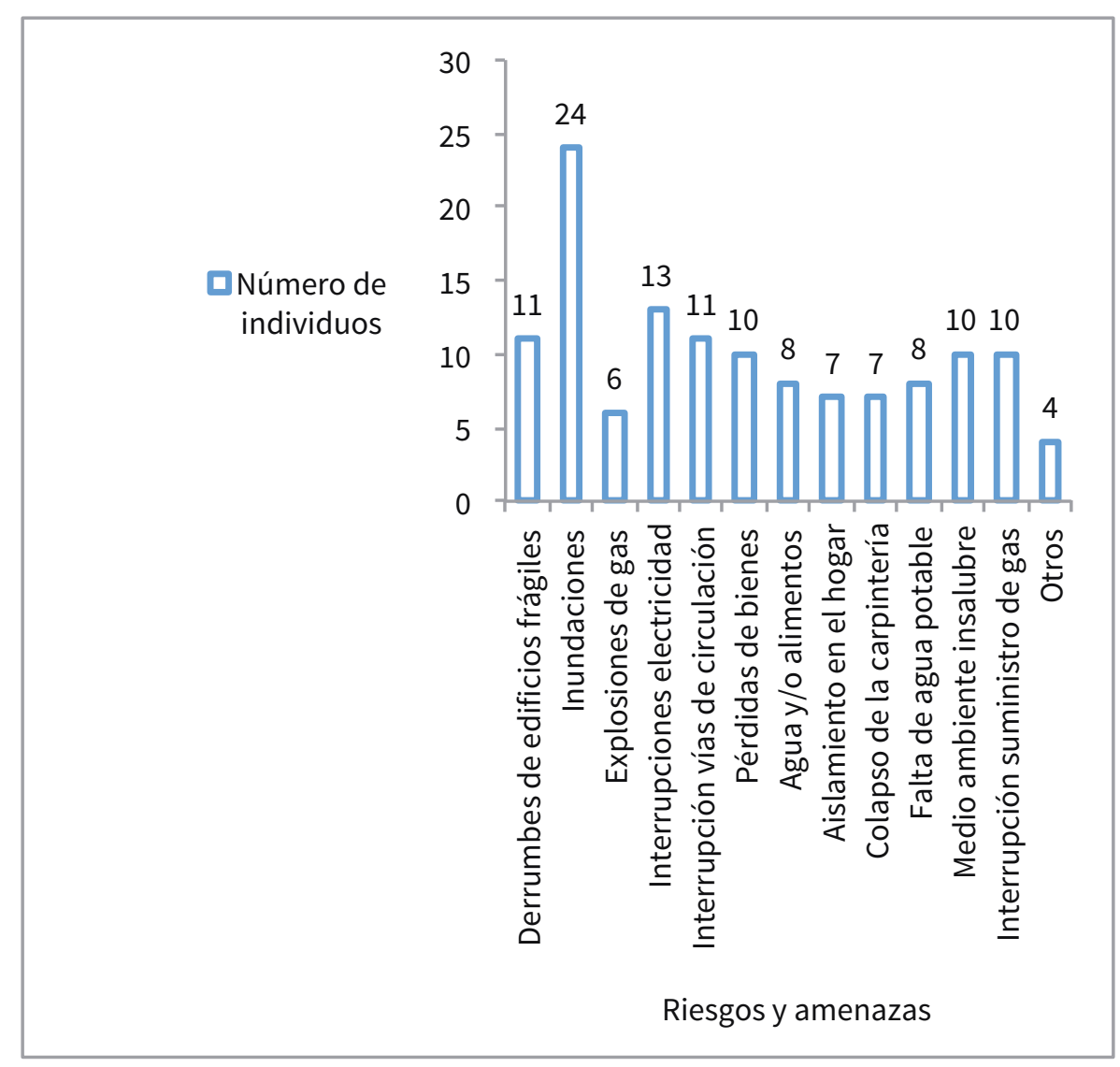

Fuente: Elaboración propia a partir de los datos del cuestionario.

Aunque la totalidad de la población consultada ha sufrido situaciones de emergencia producidas por eventos hidrometeorológicos extremos, la vivencia personal que de ellas se desprende varía entre sus habitantes. El $52,38 \%$ de la población manifestó haber vivido las situaciones de riesgo de desastre de manera traumática, y el $65,54 \%$ de los consultados asoció la palabra ciclón con términos como desastre, horror, pánico, estrés y nervios.

El problema de los "nervios", la inseguridad y el miedo en dichas ocasiones, aparece reflejado en la totalidad de los instrumentos aplicados, aunque no se prioriza en los planes de respuesta, según asegura el psicólogo en desastres, Dr. Alexis Lorenzo, y, sin embargo, obstaculiza seriamente las tareas de reducción del riesgo implementadas durante la respuesta, afirma la coordinadora de CDR.

Finalmente, la familiaridad de la población estudiada, al embate de ciclones y fuertes lluvias, se traduce en un exceso de confianza ante situaciones de riesgo de desastre, generando escenarios de indisciplina ciudadana, lo cual constituye uno de los principales problemas de la comunidad detectados por los trabajadores sociales y la autora del Programa de Comunicación Social para Desastres, Inola Díaz. 


\section{Conocimiento, autopercepción del conocimiento y predisposición a formarse}

Labrada afirma que "muchos cubanos siguen lo que ocurre en las noticias cuando se aproxima un ciclón pero se confían porque creen saber más de lo que saben". Esta afirmación se confirma en el cuestionario. Aunque la población encuestada posee un adecuado nivel de conocimiento acerca de mitos y realidades en torno a los desastres (ver gráfico 2), la población encuestada cree saber más de lo que realmente conoce (ver tabla 2).

\section{Gráfico 2. Calificaciones obtenidas sobre el conocimiento de desastres de origen hidrometeorológico extremo}

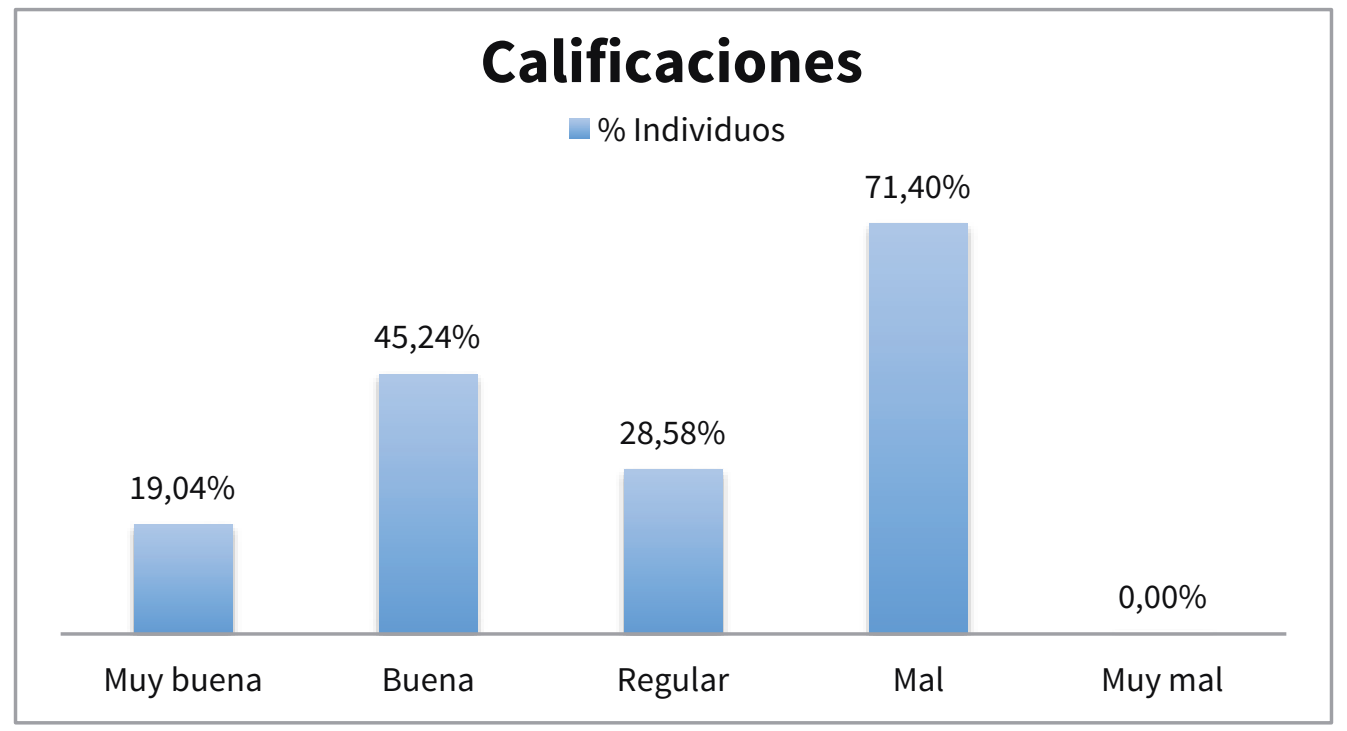

Fuente: Elaboración propia a partir de los datos del cuestionario.

Leyva, de la Defensa Civil, reconoce la necesidad de seguir "trabajando en el sentido de la percepción del riesgo, porque hay personas caprichosas, incrédulas, que no siempre tienen el deseo de asumir con responsabilidad lo que está ocurriendo en un momento".

Tabla 2. Cantidades relativas a la autopercepción de conocimiento sobre DN

\begin{tabular}{|l|l|}
\hline \multicolumn{1}{|c|}{ Categoría } & \multicolumn{1}{c|}{$\%$} \\
\hline Muy informado & 44,18 \\
\hline Informado & 44,19 \\
\hline Medianamente informado & 6,98 \\
\hline Poco informado & 2,33 \\
\hline Nada informado & 2,33 \\
\hline
\end{tabular}

Fuente: Elaboración propia a partir de los datos del cuestionario. 
Desde el municipio se han creado espacios para trabajar asuntos relevantes para la población relacionados, entre otros temas, con la prevención de riesgos de desastres, sin embargo, según informaron diversos actores comunitarios, apenas asiste la vecindad: "algunos sí, pero es la minoría. Otros son muy incrédulos porque no creen en lo que uno dice, o creen saberlo todo, entonces no asisten, se mantienen así de lejos" admite Liudmila. La coordinadora de CDR, Ana María, confirma también este desinterés cuando afirma que "a la población le es indiferente porque siente que es volver a escuchar lo mismo que han escuchado en otras ocasiones y finalmente acuden las personas que menos lo necesitan porque son las más sensibilizadas y conscientes con el tema". La exdelegada de la circunscripción igualmente reconoce que los vecinos de la zona № 62 "no contestan al llamado y no participa la masividad que desearían, contrariamente, la población que asiste es la mínima”.

Los líderes formales en todos sus niveles, satisfechos con difundir la información que les es enviada desde instancias superiores, visualizan la desmotivación ciudadana sin asumir su responsabilidad y la atribuyen a la apatía general de la población, declara un presidente de CDR. Únicamente la coordinadora del CDR entrevistada aceptó con humildad su responsabilidad debido a una deficiente estrategia que no consigue llegar a su público, "no hemos logrado que la gente sienta que esto forma parte de sus vidas, de que le estamos cuidando sus vidas", admite Ana María.

\section{Medios de comunicación y líderes informales}

La población consultada concede una gran fiabilidad a la información difundida por la televisión, radio y prensa. El $88,09 \%$ de la muestra cree que el papel de los medios de comunicación es incuestionable; lo que se dice en ellos se acepta de forma tajante e irrefutable.

La noche del 2 de marzo de 2010, la televisión cubana pronosticó penetraciones de carácter leve en el litoral de La Habana. No obstante, estas penetraciones causaron fuertes inundaciones y cuantiosos daños en El Vedado-Malecón, lo que causó la indignación de los vecinos. Lo que sucedió, explica el ingeniero hidráulico y experto en desastres, Jorge Luis Rodríguez Viera, fue que la audiencia asumió la información ofrecida por la televisión y obvió los elementos vulnerables del barrio (léase el mal estado del alcantarillado que no sirve como tragante o la existencia de viviendas por debajo del nivel de las calles). La penetración ciertamente fue de carácter leve pero sus efectos negativos se multiplicaron al converger con el lamentable estado arquitectónico de algunas viviendas en la parte baja del barrio, cuestión que no había sido alertada, ni tratada por la televisión, ni por el resto de los medios que se habían limitado a describir la fuerza del evento físico.

Además de la confianza que la población deposita en los medios de comunicación, estos siguen siendo los canales principales a través de los cuales los habaneros del área estudiada consideran haber obtenido mayor conocimiento en materia de prevención de desastres. Radio, televisión y prensa aparecen como las vías prioritarias de aprendizaje (ver Gráfico 3), incluso son percibidos como más eficaces que el aprendizaje adquirido por la propia experiencia empírica en pasados eventos. 


\section{Gráfico 3. Porcentaje de las vías de información/formación prioritarias de la población estudiada}

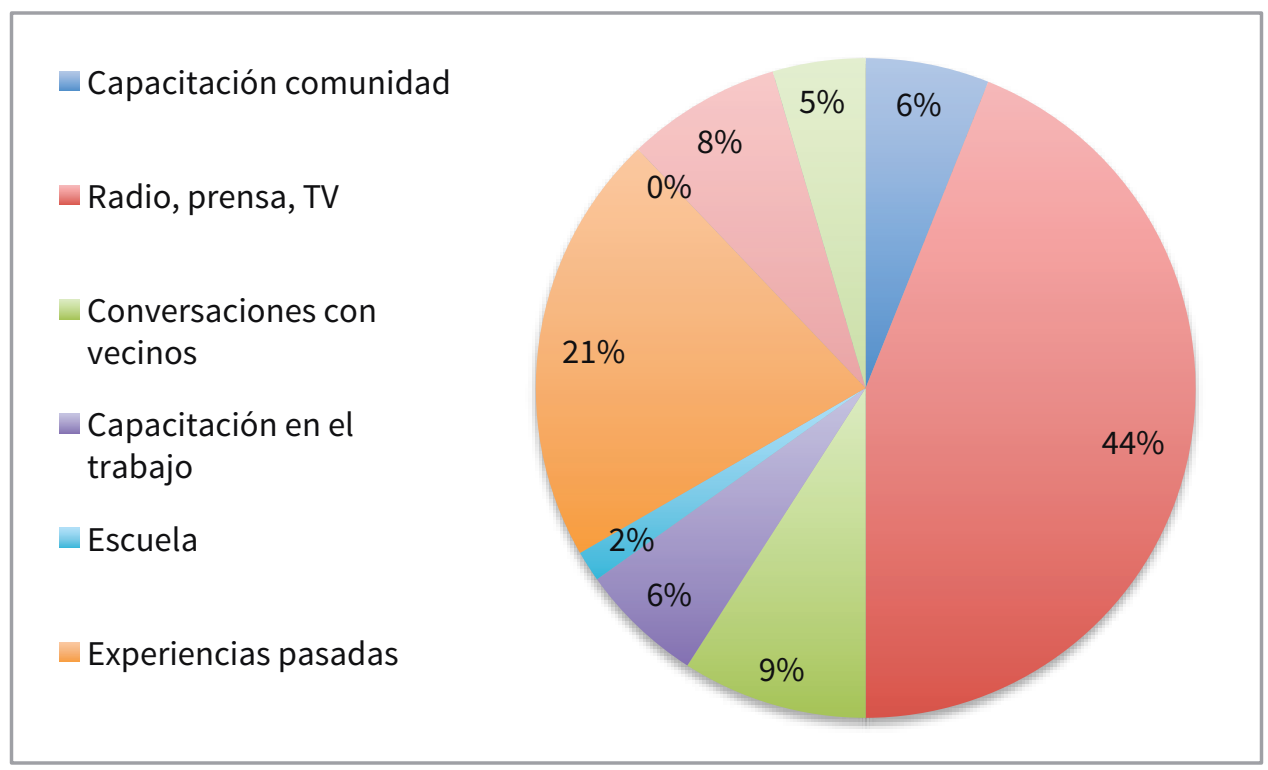

Fuente: Elaboración propia a partir de los datos del cuestionario.

Tras los medios de comunicación y la experiencia personal, los líderes informales de la zona objeto de estudio se aproximan a la temática de prevención de riesgo de desastres en los intercambios simbólicos que se producen como consecuencia de las interrelaciones que se fraguan a través del diálogo con otros vecinos de la circunscripción, léase reuniones de CDR, intercambios espontáneos de vecinos, Día de la Defensa. Un dato que llama la atención es que, en la época tecnológica actual, ninguno de los encuestados menciona internet como una vía de información prioritaria ${ }^{30}$.

\section{Contenidos priorizados}

Los contenidos generales que proponen las estrategias de comunicación para la prevención de desastres a nivel nacional e institucional engloban prácticamente la totalidad de las inquietudes de los encuestados, sin embargo, las divergencias aparecen cuando la estrategia aterriza en la localidad. Los programas educativos prescritos desde el municipio no siempre coinciden con las motivaciones de los habaneros de El Vedado-Malecón, y obvian una gran variedad temática que pudiera favorecer su asistencia y participación en estos intercambios comunicativos.

A los líderes informales (ver gráfico 4) de la zona estudiada les concierne principalmente los asuntos relacionados con las intervenciones constructivas en el hogar (como muretes o "sobrepisos") que ayuden a salvar los bienes durante inundaciones; es curioso el interés que existe - en igual medida que la anterior - por la creación de un plan familiar que sirva de guía para minimizar los daños ante un evento riesgoso.

30 Cuba es uno de los países con menor tasa de conectividad a internet (Efe, 2015). 
Gráfico 4. Temáticas que interesan a los líderes informales

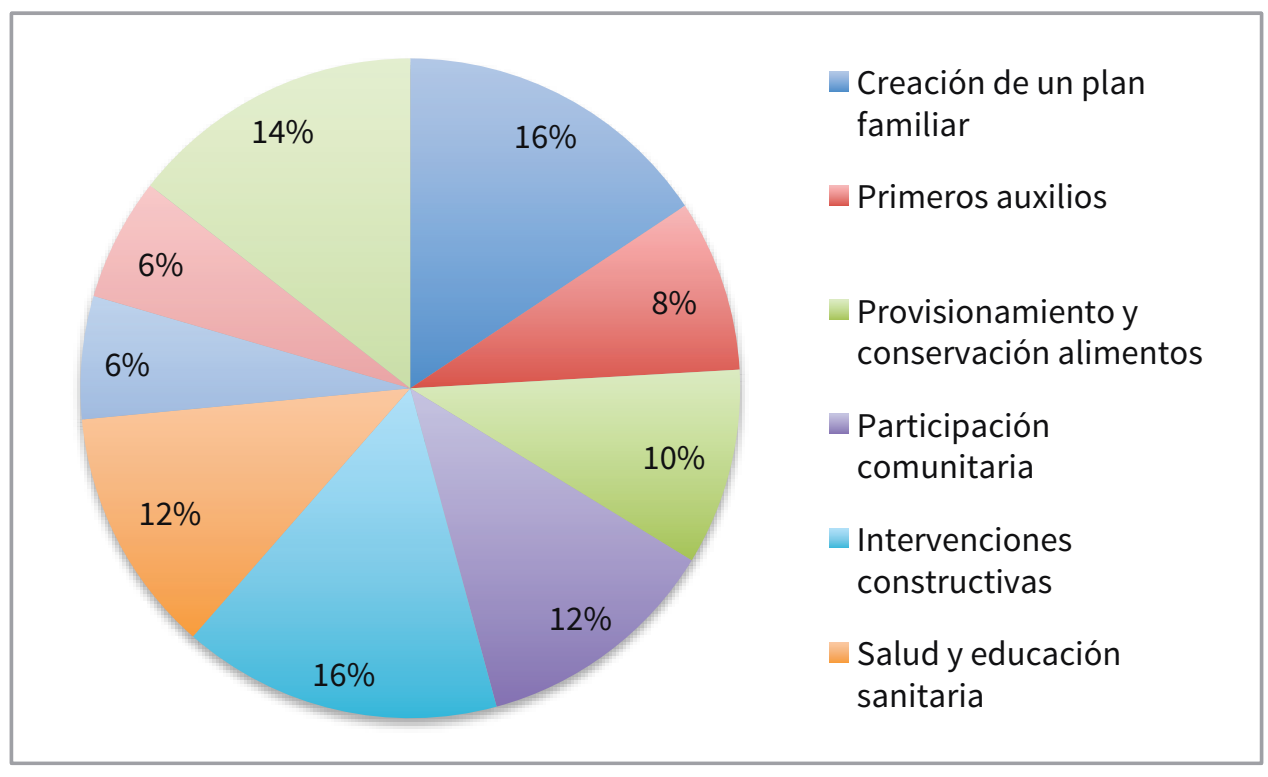

Fuente: Elaboración propia a partir de los datos del cuestionario.

El tercer bloque temático que más preocupa a los habitantes de la circunscripción es la vuelta a la "normalidad" lo antes posible, es decir, la recuperación tras el impacto. En adición a lo anterior, se recogieron en el cuestionario sugerencias y observaciones que reflejan el deseo y la necesidad de desarrollar un ejercicio de preparación para casos de desastres similar al "Meteoro ${ }^{31}$ " en el nivel comunitario y no solo circunscrito a los centros de trabajo.

\section{Actitud hacia la toma de medidas preventivas}

El número de líderes que de manera organizada posee en el hogar un plan familiar para situaciones de desastre es muy limitado, solo el 23,81\% de la muestra asegura tener uno (ver gráfico 5), siendo este uno de los contenidos preferidos (ver gráfico 4) por los líderes estudiados. La ausencia de planificación en situaciones de desastre propicia, ante la llegada de un ciclón u otro evento adverso, la ejecución de medidas de forma reactiva, ciertamente sobre la base del conocimiento y la experiencia, pero que no dejan de tener un matiz espontáneo y con ciertas dosis de improvisación, en muchas ocasiones mediadas por el estrés y la ansiedad que se genera en esos momentos de crisis.

31 Cada mes de mayo, justo antes del comienzo de la temporada ciclónica se realiza un ejercicio de entrenamiento de dos días de duración, conocido como Meteoro, para preparar a la población ante los peligros naturales. 


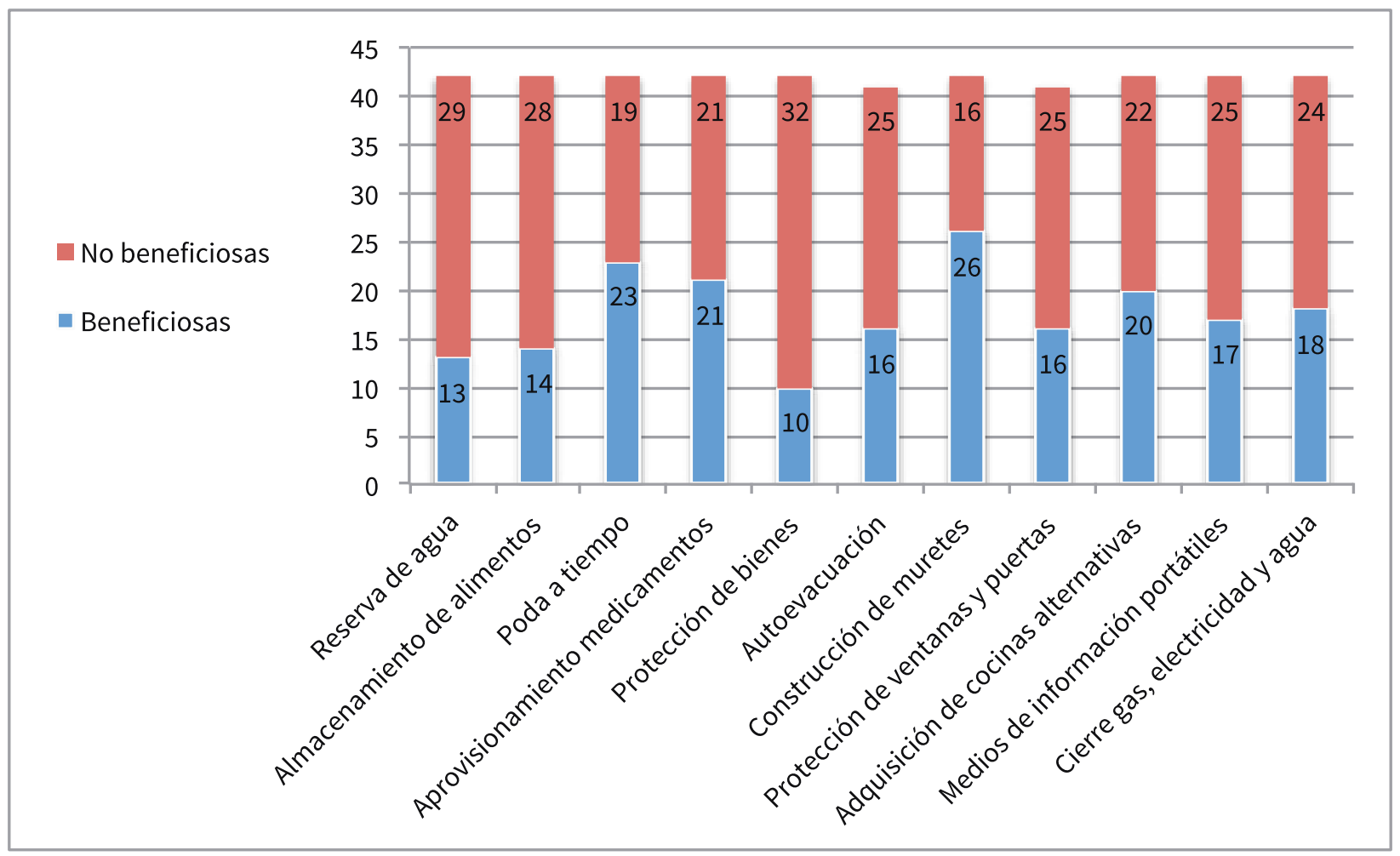

Fuente: Elaboración propia a partir de los datos del cuestionario.

En cuanto a la predisposición para evacuar, si bien una gran parte de la población está dispuesta a movilizarse en caso necesario, existe un 34,88 \% que todavía se muestra indecisa (ver tabla 3).

\section{Tabla 3. Predisposición a evacuar}

\begin{tabular}{|l|l|}
\hline \multicolumn{1}{|c|}{ Categoría } & \multicolumn{1}{c|}{$\%$} \\
\hline Dispuesto & 58,14 \\
\hline No dispuesto & 6,97 \\
\hline Tal vez & 34,88 \\
\hline
\end{tabular}

Fuente: Elaboración propia a partir de los datos del cuestionario.

Este porcentaje significativo constituye los posibles casos de resistencia a abandonar sus hogares, entorpeciendo las tareas de evacuación ante la inminente presencia de un ciclón tropical. Si bien las fuerzas armadas cubanas pueden actuar por la fuerza en caso de máxima oposición a evacuar, los trabajadores de la comunidad 
aseguran que nunca es necesario acudir a la fuerza, ya que finalmente las personas son "convencidas" y acceden a abandonar la casa, declara Xiomara, "aunque el proceso de evacuación se ralentiza".

La Dra. Virginia Huergo, de la Cruz Roja Cubana, confirma esta idea: "la gente puede saber mucho qué es un ciclón pero cuando viene, uno de los grandes trabajos es concienciarlos para sacarlos de sus casas y eso es bastante difícil". El Dr. Calviño ${ }^{32}$ mantiene una posición similar a la de la doctora: por mucho conocimiento que se haya acumulado sobre un tema de esta índole -opina el profesor- el momento de la decisión final "no te lo quita nadie de encima. En el proceso de toma de decisiones, los medios masivos se quedan cortos y tienen que ser los medios de corta distancia los que trabajen".

La medida preventiva considerada como la más beneficiosa por los líderes informales y a la que más se recurre es la construcción de muretes y otras reformas en las viviendas: el 61,90 \% de la muestra asegura estar muy satisfecho con la adopción de este tipo de acciones para la prevención de riesgos de desastres. Lógicamente, recuérdese que es este (ver Gráfico 4) es uno de los contenidos preferidos en caso de asistir a una acción formativa.

Entre estas acciones positivas detectadas se encuentran las relacionadas con el almacenamiento de medicamentos $(88,09 \%)$ y alimentos $(85,71 \%)$, la reserva de agua necesaria $(71,42 \%)$ y la correcta protección de puertas y cristales $(78,57 \%)$.

\section{Conclusiones}

\section{Acerca de los líderes formales}

a. Los máximos responsables de hacer llegar los lineamientos marcados en los planes nacionales a las comunidades son los líderes formales, figura reconocida y aceptada por la población.

b. La cercanía que mantienen con la comunidad es fundamental en los procesos de RRD, ya que genera confianza y favorece la gobernabilidad en cuestiones de reducción del riesgo de desastres, lo que permite salvaguardar limitaciones tan serias como las dificultades comunicativas. Dicha proximidad les otorga legitimidad para definir objetivos y diseñar intervenciones específicas que favorezcan la reducción de desastres.

c. No obstante, su grado de familiaridad y conocimiento de las necesidades locales no es aprovechado en toda su potencialidad desde instancias superiores.

\section{Acerca de los líderes informales}

d. Las mayores posibilidades de éxito en las intervenciones para la RRD de los líderes formales es mediante el empleo de los líderes informales como interlocutores de las mismas por su potencial de diseminadores de la información y generadores de confianza.

32 Emblemático presentador de la televisión cubana. 
e. El potencial de la mujer en espacios locales en asuntos referidos a la RRD por su responsabilidad, empatía y constancia en las labores comunitarias, y el sentimiento generalizado de la existencia de lazos de solidaridad, confianza e incondicionalidad son fortalezas a tener en cuenta en el contexto estudiado.

f. A pesar del nivel de conciencia y conocimiento sobre riesgos de desastres de origen hidrometeorológico extremo alcanzados en el área estudiada, existe un universo de mitos en torno a los desastres que generan percepciones sesgadas y estereotipadas. El más generalizado es el que identifica los eventos climatológicos extremos -como huracanes-con desastres naturales.

g. En general, los líderes estudiados conocen los peligros climatológicos que les amenazan pero desconocen el grado de vulnerabilidad en el que se desenvuelven ante la inminente llegada de un evento climatológico adverso, lo que propicia un estado de "confianza" y "relajación" que afecta la toma de medidas preventivas "a tiempo", en especial aquellas que contribuyen a reducir vulnerabilidades.

\section{Elementos que influyen en la percepción del riesgo}

h. Junto al elemento sorpresa, las falsas creencias, el estrés y el miedo que generan las situaciones de desastres, la familiaridad y la confianza de los sujetos estudiados debido a la exposición constante a eventos hidrometeorológicos extremos son algunos motivos detectados que distorsionan la percepción del riesgo, la concienciación y la toma de medidas preventivas ante situaciones riesgosas.

i. Una vez más se verifica el rol de los medios de comunicación como agentes socializadores en la reducción de desastres, con una responsabilidad indiscutible en su representación social y la del riesgo de desastre y su percepción. La confianza depositada en los medios produce una serie de consecuencias: moldea la percepción social del riesgo; permite ofrecer instrucciones e informar de los acontecimientos con la seguridad de que las audiencias confían en lo que se les comunica, aumentando la probabilidad de obedecer; pero al mismo tiempo, reduce la capacidad de reflexión de los interlocutores, sin dejar margen a la crítica y a la interpretación.

\section{Acerca de las intervenciones}

j. Las contradicciones en los modos de entender la prevención y la preparación para desastres, ciertas deficiencias comunicativas, la inadecuación de los contenidos a los intereses de los sujetos y la sobreelevada percepción de conocimiento son elementos detectados que no son tenidos en cuenta desde los niveles superiores, reducen la efectividad de las intervenciones de preparación comunitaria y explican la desmotivación que muestra la comunidad a la hora de participar en dichas intervenciones.

k. Prevalece entre los responsables de dichas intervenciones una mirada ajena al problema en el sentido de que ellos se sienten satisfechos porque presuponen el hecho de que al enviar la información, esta llega a los sujetos y es además comprendida. Este entender evidencia las disposiciones históricas de los procesos formativos e informativos basados en concepciones eminentemente unidireccionales y pone de relieve la dificultad para superar dichas concepciones. 


\section{A modo de síntesis final}

Las constantes amenazas y frecuentes inundaciones que sufre el barrio estudiado, el mal estado constructivo de algunas de las viviendas y las acusadas desigualdades sociales producen una visión fatalista del desastre que hace priorizar entre sus líderes las intervenciones de respuesta. Los contenidos que se dirigen a mejorar la respuesta (como la construcción de barreras protectoras) son los preferidos por la comunidad y constituyen para ellos las medidas preventivas más beneficiosas.

No es de extrañar, con lo dicho anteriormente, que para la población estudiada, en especial la más vulnerable, la reducción de desastres tiene más que ver con prepararse para la respuesta a los desastres que con la lógica de reducción de vulnerabilidades, ya que aunque se perciben las amenazas, no se perciben de igual manera las vulnerabilidades.

\section{Referencias}

1. Almaguer Riverón, C. D. (2008). El riesgo de desastres: una reflexión filosófica. Tesis en opción al grado de doctor. La Habana: Universidad de La Habana.

2. Arias Herrero, H. (s.f). Estudio de las comunidades. En P. De la Torriente. (Ed.), Lecturas sobre comunicación en la comunidad. La Habana.

3. Arias Herrero, H. Consejos Populares: estructura comunitaria. En P. De la Torrienre. (Ed.), Lecturas sobre comunicación en la comunidad. La Habana.

4. Baccin, C. (2001). Diagnóstico de comunicación. Desafíos del "venir entre". Signo y Pensamiento XX.

5. Balanovsky, V. Redin, M.E., y Pogiesse, H.. (2001). Percepción social del riesgo inundaciones en el arroyo Maldonado: mapa de riesgo elaborado con participación comunitaria. La Red de Gestión Asociada del Oeste, a solicitud del Banco Mundial.

6. Beltrán, L. R. (2004). Comunicación educativa e información pública sobre desastres en América Latina: Notas para reflexionar. Antigua: UNESCO, EIRD, Federación Internacional de la Cruz Roja, OPS/OMS.

7. Caballero Pérez, Y. (2010). Caracterización detallada del Consejo Popular Vedado-Malecón. La Habana: Programa de trabajadores sociales Plaza de la Revolución.

8. Castellanos Pérez, A. (2009). Esencia de la reducción de desastres. Vías para lograr la reducción de vulnerabilidades. En Salud y desastres. Experiencia cubana. La Habana: CLAMED.

9. Cuba. (1997). Decreto Ley № 170 del Sistema de Medidas de la Defensa Civil de la República de Cuba, firmada por Fidel Castro Ruz. La Habana.

10. Cuba. (2005). Directiva №1 del 1 de junio de 2005 del Vicepresidente del Consejo de Defensa Nacional para la Planificación, Organización y Preparación del País para las Situaciones de Desastres. Gaceta Oficial de la República de Cuba. Firmada. por Raúl Castro Ruz. La Habana.

11. DCC. (2006). Programa de comunicación social para la reducción de desastres en Cuba. La Habana: Editora Política.

12. EFE. (2015). Crece la conectividad a internet en Cuba. La Habana: Recuperado de http://cartasdesdecuba. com/crece-la-conectividad-a-internet-en-cuba/ 
13. Farré Coma, J. y Gonzalo Iglesia, J. L. (2009). Discursos y prácticas en el proceso de comunicación de riesgo petroquímico. En C. Moreno Castro, Comunicar los riesgos. Ciencia y tecnología en la sociedad de la información. Madrid: Ed. Biblioteca Nueva OEI.

14. Fischoff, B. (1995). Risk Perception and Communication Unplugged: Twenty Years of Process. Society for Risk Analysis.

15. Gaeta, N. (2016). Ciudad sostenible, participación sostenible y prevención de desastres. Publicación presentada en el IV Congreso Internacional Ciudades Creativas. Madrid.

16. Gaeta, N. y Lorenzo, A. (2012). La evacuación masiva. Su abordaje desde las relaciones de confianza entre autoridades y sociedad civil. Prisma Social No 8. La incertidumbre Social, 116-150.

17. Hollander, E. P. (1967) Principios y métodos de la psicología social. Oxford University Press: Oxford.

18. Lavell, A. (1996). Degradación ambiental, riesgo y desastre urbano: problemas y conceptos. En M. A. Fernández, Ciudades en riesgo. Lima: La Red/USAID.

19. Lavell, A. (2000). Desastres y desarrollo: Hacia un entendimiento de las formas de construcción social de un desastre: El caso del Huracán Mitch en Centroamérica. En N. Garita y J, Nowalski, Del desastre al desarrollo sostenible: Huracán Mitch en Centroamérica. BID-CIDHS. Ginebra.

20. Llanes Guerra, J. y Grupo Excelencia (2008). Cuba. Paradigma en la reducción del riesgo de desastres. La Habana: OXFAM Solidaridad.López Cerezo, J. A. y Luján, J. L. (2000). Ciencia y política del riesgo. Madrid: Alianza Editorial, D. L.

21. Luhmann, N. (1998). Sociología del riesgo. México: Universidad Iberoamericana.

22. Mitrani Arenal, I. et. al. (2000). Las penetraciones de mar en las costas de Cuba. Las zonas más expuestas y su sensibilidad al cambio climático. Informe de investigación. La Habana: CITMA e Instituto de Meteorología de Cuba.

23. Navarro Machado, V. R. (2009). Situaciones de desastres. Manual para la preparación comunitaria. La Habana: Editorial Ciencias Médicas.

24. PNUD/ONU. (2004). La reducción de riesgos de desastres un desafío para el desarrollo. Nueva York: Informe Mundial del PNUD.

25. Portal Moreno, R. (2003). Por los caminos de la utopía. Un estudio de las prácticas comunicativas de los Talleres de Transformación Integral del Barrio en la ciudad de La Habana. Tesis presentada en opción al grado científico de Doctora en Ciencias de la Comunicación. La Habana: Universidad de La Habana.

26. Puertas López, E. (2003). Análisis psicosocial sobre percepción del riesgo y actitud hacia la información de la población afectada por planes de emergencia nuclear. Madrid: Dirección General de Protección Civil.

27. Rodríguez Viera, J. L. (2009). Lo vedado de El Vedado. Herramientas metodológicas. Ejemplos y caso de estudios para la gestión estratégica integral de riesgos urbanos integrados. La Habana: Sello Editoria GDIC.

28. Slovic, P. y Weber, E. (2002). Perception of Risk Posed by Extreme Events. Center for Decision Sciences (CDS) Working Paper. Columbia University. New York.

29. Slovic, P., Fischhoff, B. y Lichtenstein, S. (1981). Facts and Fears: Societal Perception of Risk, NA - Advances in Consumer Research 8.

30. Thompson, M. y Gaviria, I. (2004). Cuba, superando la tormenta: lecciones de reducción del riesgo en Cuba. La Habana: OXFAM América. 
31. UNISDR Panamá. (2009). Terminología sobre reducción del riesgo de desastres. Ginebra:

32. VV.AA. (s.f). Mitos y realidades sobre desastres. La Habana: Centro Latinoamericano de Medicina de desastres.

33. White, G. F. (1945). Human Adjustment to Floods. Department of Geography Research Paper.

34. Wilches-Chaux, G. (2001). Sobre "aguaceros" y "goteras". Bogotá. Recuperado de http://enosaquiwilches. blogspot.com

35. Wilches-Chaux, G. (1989). Fundamentos éticos de la gestión del riesgo. Bogotá. Recuperado de http:// www.ucentral.edu.co/NOMADAS/nunme-ante/21-25/nomadas-22/4-gustavo\%20fundamentos-ok.pdf 